\title{
Image-Based Characterization of Alternative Fuel Combustion with Multi-Fuel Burners
}

\author{
Markus Vogelbacher, Patrick Waibel, Jörg Matthes and Hubert B. Keller
}

\begin{abstract}
Many industrial high-temperature processes such as e.g. cement production employ multi-fuel burners in order to achieve the required energy input with low-cost alternative fuel. So far, a constant operation of multi-fuel burners with high fractions of alternative fuel $(>\mathbf{7 0 \%})$ is not possible due to inherent fluctuating fuel properties. Energy input and product quality are directly affected by varying points of combustion time, different scattering of fuel and insertion of unburned fuel or chemical substances into the product. We propose an image processing system based on infrared images that detects the alternative fuel streakline and derives parameters for the characterization of the flight and burning behavior. Using these parameters, an adjustment of the burner settings depending on the fluctuating fuel properties can be carried out. This automatic monitoring and control of the combustion process allows an increased use of alternative fuels in constant operation. Experimental data from a rotary kiln for cement clinker production are used to validate the image processing system.
\end{abstract}

Index Terms - cement industry, fuels, image processing, infrared image sensors, process control.

\section{INTRODUCTION}

I $\mathrm{N}$ the field of industrial combustion processes the number of multi-fuel burners has increased significantly during the last years. These multi-fuel burners can use alternative fuels like plastic, tire chips or animal meal in arbitrary high fractions besides fossil primary fuels such as brown coal. Due to declining availability of fossil fuels, the use of these lowcost, low-ranked and in large quantities available alternative fuels provides a great savings potential. At the same time the increased use of alternative fuels can not only reduce costs but also reduce emissions [1]. Fig. 1 shows an example of such a multi-fuel burner that includes different supply channels for fuel and combustion air.

Because of differences in both the physical and chemical properties as well as in the reaction behavior, alternative fuels differ in their combustion characteristics basically from fossil fuels. Furthermore, there are also differences in the combustion behavior within the different alternative fuels. This is mainly influenced by the quality of the fuels and the associated fluctuations of the properties. Such properties can be e.g. the humidity or the particle size, which have a significant influence on the flight characteristics and the combustion time of the fuel. Accordingly, the fluctuations of the fuel properties determine the entire combustion process and by adding unburned fuel to the product also the product quality

M. Vogelbacher, P. Waibel, J. Matthes and H.B. Keller are with the Institute for Applied Computer Science, Karlsruhe Institute of Technology (KIT), Hermann-von-Helmholtz-Platz 1, 76344 Eggenstein-Leopoldshafen, Germany e-mail: markus.vogelbacher@kit.edu. is directly influenced. For this reason, a constant operation of multi-fuel burners with a high fraction of alternative fuel compared to the total fuel $(>70 \%)$ is not possible so far. To further increase the fraction of alternative fuel at a constant operation, it is necessary to permanently monitor the fuel behavior and control the burner or process parameters. With currently used measurement systems this continuous monitoring is not possible:

- The evaluation of laboratory samples of the final product is time-delayed and does not allow fast adaptions of the process control system.

- In images which are acquired by current camera technologies for process monitoring (sensitive in the visual or near-infrared wavelength range) the alternative fuel is occluded by the flame and therefore not visible. Conclusions on the burning behavior of the alternative fuel are not possible with these systems.

The continuous monitoring and image-based characterization of alternative fuel combustion with multi-fuel burners represents a new challenge. In this work a method for the detection of the average streakline of the alternative fuel based on a long-wavelength infrared camera system is described. The robust detection of the average streakline forms a basis for the calculation of further features that describe the burning behavior of the alternative fuel such as the point of impact, the exit angle at the burner mouth, the point of combustion and the scattering. How these image-based features can contribute to enhance the overall industrial process is exemplary shown by means of a potential control framework that takes into account the point of impact of unburned fuel. The development and the validation of the average streakline detection method was conducted with data that has been acquired at an industrial rotary kiln for cement clinker production. During a two-day measurement campaign, actuating variables of the process have been changed to cover a large range of different process states in the image data.

\section{A. Related work}

Image processing techniques can be used to characterize the flame appearance or the fuel behavior. In order to obtain analyzable image data, it is necessary to select a suitable camera system. Amiruddin and Sapuan [2] show some RGB and false-color images of a burner flame at an industrial combustion process. Ballester and Garcia-Armingol [3] provide an overview of available sensor systems for flame monitoring. Baek et al. [4] and Horng et al. [5] evaluate the combustion processes through the transformation of the flame images to 
the HSI color space. The evaluation of the process and the comparison with process parameters is only based on HSI color values. Duchesne et al. [6] and Yu and MacGregor [7] use multivariate image analysis and data analysis techniques to derive different flame properties that can be associated with process parameters. The work of $\mathrm{Lu}$ et al. [8], Chen et al. [9], Smart et al. [10] and Sun et al. [11] deal with the flame characterization on the basis of camera systems in the visual wavelength range and information of photo-detectors. With the help of image processing, frequency analysis and data analysis methods, geometric parameters (e.g. flame size, shape), luminescent parameters (e.g. brightness) and thermodynamic parameters (e.g. flame flicker, temperature) can be obtained. The methods mainly consider the flame shape and therefore not directly the individual fuel components. Yang et al. [12], Zhang et al. [13] and Matthes et al. [14] detect and track individual fuel particles within the flame. Monitoring the fuel behavior using an infrared camera system and subsequent image analysis can not be found in literature. For other fields of application of rotary kiln processes such as special waste incineration or zinc recycling [15]-[17], different information can be derived out of infrared images e. g. from the segmented solid bed. Zipser et al. [18] provide a system for camera-based analysis of industrial combustion processes. The detection and utilization of streaklines in images is not very common. In [19] streaklines are proposed as a representation of flow in crowded scenes.

\section{B. Paper outline}

In Section II an overview of multi-fuel burners, infrared camera systems, image processing and the experimental setup is given. New methods for estimating the alternative fuel streakline are presented in Section III. Section IV includes the generation of reference data and the comparison to the results of the new methods. Additionally, a control framework is presented which exemplary shows the applicability of the image-based features in the process control system. A conclusion and an outlook are given in Section V.

\section{BASICS FOR IMAGE PROCESSING AT MULTI-FUEL BURNERS}

In this section the structure of a multi-fuel burner and explanations on the use of infrared cameras for combustion processes are presented. Furthermore, an overview of the image processing work-flow and the experimental setup is given.

\section{A. Multi-fuel burner}

As shown in Fig. 1, a multi-fuel burner consists of different supply channels for fuel and combustion air. Fig. 2 shows the structure of a multi-fuel burner, which is used in the application of this work. The primary fossil fuel is added by the co-axially arranged coal channel. For alternative fuel, two supply channels are available. In this application, a mixture of plastic and tire chips are fed to channel 1 and animal meal to channel 2 . The flame shape can be influenced by the co-axially

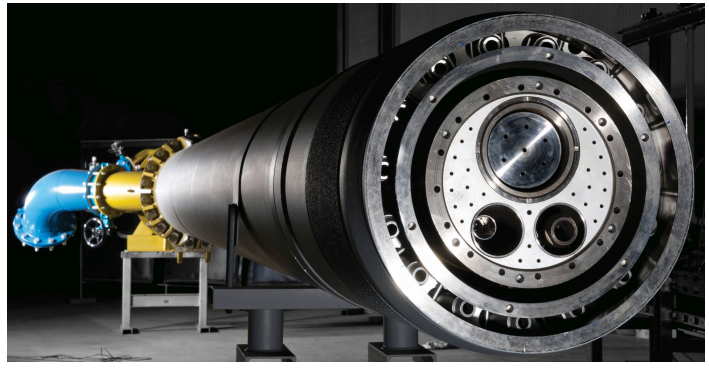

Fig. 1. Example of a multi-fuel burner from the company Unitherm Cemcon [20].

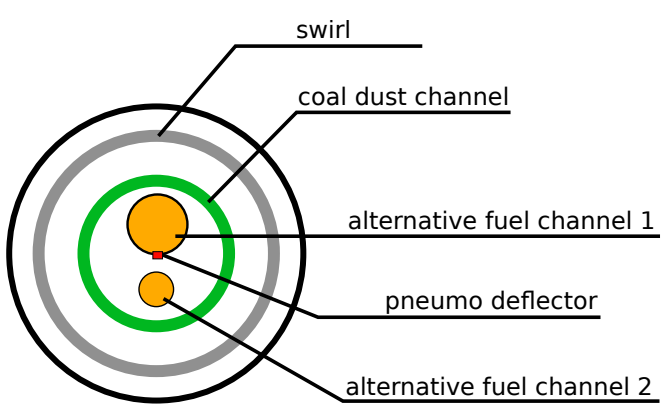

Fig. 2. Schematic description of the front of a multi-fuel burner.

arranged swirl air channel. High swirl settings (corresponding to high tangential deflection angles around the burner axis) lead to a short expanded flame, low settings to a long slim flame. By varying the pressure of the swirl air, the so-called primary air, the flame shape can also be influenced. At the end of the alternative fuel channel 1 the exit angle of the alternative fuel can be influenced by the pressure of a vertical air flow, the so-called pneumo deflector. By increasing the pressure, also the exit angle and the diversification of the fuel are increased. The rotational speed of the conveying air blower controls the initial speed of the alternative fuel of channel 1 . The pressure of central air serves to stabilize the flame. Higher pressure leads to a sharp flame near to the burner mouth.

The combustion behavior of individual fuel portions can be retraced from a lateral view in Fig. 3. The animal meal is not included in the figure because of the rapid combustion close to the burner mouth, where it is usually occluded by the coal area. The alternative fuel mixture of channel 1 leaves at the center of the burner mouth and forms the fuel streakline. Depending on the quality of the fuel, the streakline is composed by particles and agglomerations of different sizes and exhibits different appearances as e.g. location or width. Furthermore, the combustion time varies with the fuel quality and the combustion process conditions. After ignition, alternative fuel particles are no longer visible in the infrared images which yields a relationship between the length of the streakline and the combustion time. The blue line in Fig. 3 describes the average fuel streakline. Close to the burner mouth the alternative fuel is surrounded by a coal area with shorter point of combustion time. 


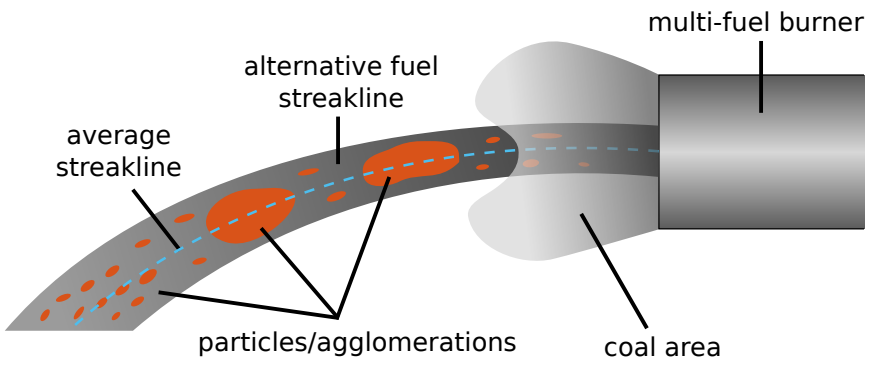

Fig. 3. Lateral schematic view of a multi-fuel burner with leaving fuel.

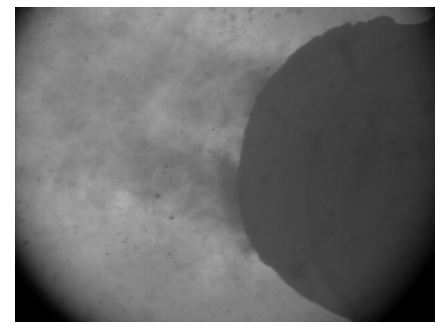

(a)

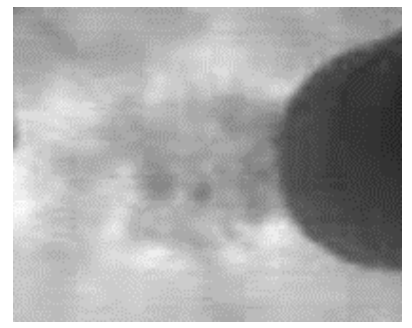

(b)

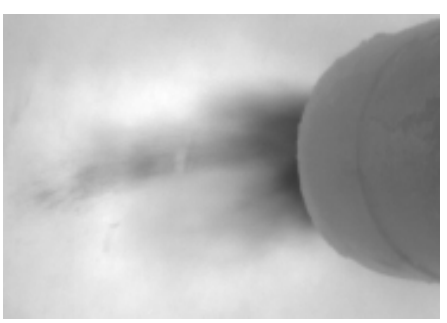

(c)

Fig. 4. Comparison of the views to a multi-fuel burner with a camera system using the (a) visual, (b) near-infrared and (c) Long-wavelength infrared range.

\section{B. Infrared camera system}

Because of the different degrees of transmission of combustion gases and solid particles, the visibility of fuel within the flame is limited and depends on the wavelength range of the camera system. Relevant combustion gases of a typical combustion process such as a rotary kiln process are water vapor $\left(\mathrm{H}_{2} \mathrm{O}\right)$, carbon dioxide $\left(\mathrm{CO}_{2}\right)$ and carbon monoxide (CO). Relevant solid particles are soot or dust. A comparison of the visibility of fuel within a burner flame of a rotary kiln for cement production with different camera systems is given in Fig. 4. The visual and near-infrared wavelength range (Fig. 4(a) and 4(b)) only allow an evaluation of the whole (opaque) flame body. In the mid-wavelength and farwavelength infrared it is possible to exploit the fact that the burning gas molecules have specific absorption spectra. In the wavelength ranges at $3.9 \mu \mathrm{m}$ and at $10.6 \mu \mathrm{m}$ the absorption for all relevant gases are low which is equivalent to a high transmission through the burning gases. Additionally, with increasing wavelength the absorption of dust and soot reduces. Consequently, these wavelengths allow a good transmission through the combustion air, soot and dust [21] and thus the visibility of fuel within the burner flame (Fig. 4(c)). For this reason, an far-wavelength infrared camera system with a sensitivity at $10.6 \pm 0.1 \mu \mathrm{m}$ has been selected.

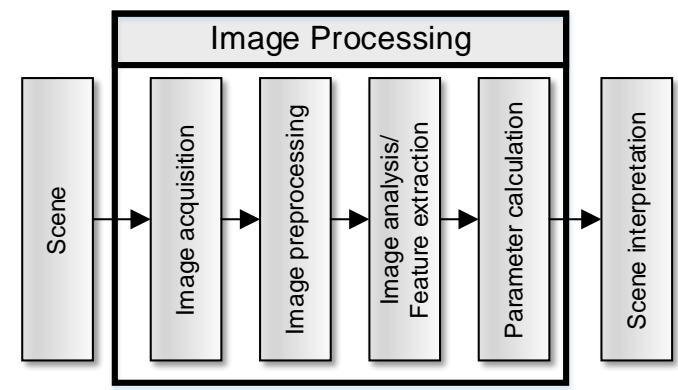

Fig. 5. Steps of image processing.

\section{Image processing}

Image processing methods can be used for automatic analysis of camera monitored scenes. The steps from image acquisition up to the interpretation of the scene can be retraced in Fig. 5. At first, the selection of an appropriate camera system that ensures the visibility of the objects of interest is important (Section II-B). In the next step, especially for low visibility conditions, it is necessary to improve the obtained raw images with preprocessing methods or to emphasize image content of interest. The subsequent analysis of the preprocessed images is thereby facilitated. Such an analysis includes for example the segmentation of particular regions, the extraction of gray value trends or also the deviation of certain image properties such as texture. Afterwards, parameters (region size, maximum gray value, etc.) can be calculated which allow an interpretation of the scene and thus enable human intervention or automatic process control.

\section{Experimental setup}

Images of a multi-fuel burner were acquired at the rotary kiln head of an industrial cement plant. The cement industry especially the area of clinker production is one of the biggest fields of application for multi-fuel burners. The thermal conversion of raw material to clinker takes place in a rotary kiln with preheater/calciner. Fluctuations in the combustion process considerably influence the conversion process and thus the product quality. Dropping and incompletely burned fuel ends up in the reaction area of the raw materials and directly affects the chemical conversion process and also the corresponding final product. A long-wavelength infrared camera (Pyroinc $380 L F$ ) with a resolution of $384 \times 288$ pixels and a frame rate of $50 \mathrm{~Hz}$ from the company DIAS Infrared was used for the image acquisition. The camera is equipped with a water cooling and air purge system that allows an operation directly inside the burning chamber. During the maintenance of the kiln an suitable opening for the camera probe lens head has been integrated at the kiln and a camera mounting has been installed.

Different burner settings for the pressure of the pneumo deflector, the rotational speed of the conveying air blower, the swirl, the pressure of primary and central air and the fuel compositions were recorded. Example images for different burner settings are shown in Fig. 6. In Fig. 6(a-b) variations of pneumo deflector and in Fig. 6(c-d) variations of swirl were 
executed. The gray values of the obtained images correspond directly with the appropriate temperatures.

\section{NEW METHOdS FOR ALTERNATIVE FUEL STREAKLINE DETECTION}

As mentioned in Section II-B, the alternative fuel can be observed in the infrared images. In this section the procedure for automatic detection of the alternative fuel is presented. Due to the concentrated output at the burner mouth a dark closed tail of fuel arises in this area. Further away from the burner, the fuel scatters more and more and individual fuel particles of different sizes and agglomerations are observable. Accordingly, the two different appearances of alternative fuel are considered with two detection methods. The combination consolidates the advantages of both methods.

\section{A. Average fuel streakline}

As basis for the extraction of further image-based parameters, the authors propose a robust detection of the average fuel streakline. The tracking of single particles over time is not a robust approach in this industrial application (the fuel particles' appearances change a lot from frame to frame). Also, optical flow methods result in very unsatisfactory flow fields due to strong disturbances in the image data which are caused by the monitoring of a harsh process environment. Streaklines are the loci of points of all particles which originated from the same initial position. Considering multi-fuel burners, all fuel particles are introduced into the kiln from the burner mouth which represents the initial position in the images. With the large amount of fuel particles an average streakline can be determined. This average fuel streakline allows e.g. the calculation of further features such as the point of impact of alternative fuel, the point of combustion or the width/scattering of the alternative fuel. A first evaluation of the fuel streakline is obtained by detecting the dark tail of the alternative fuel close to the burner mouth. An overview of the method for evaluation of this tail is shown in Fig. 7.

An input image is described by the gray values $g(x, y, n)$ at position $x, y$ and frame number $n$. In the first step, this input image $g(x, y, n)$ is cropped to a region of interest (ROI), where fuel can occur (Fig. 8(b)). Since the average streakline is of interest, a recursive time averaging algorithm is applied (Fig. 8(c)).

$$
g_{\text {mean }}(x, y, n)=\frac{n-1}{n} g_{\text {mean }}(x, y, n-1)+\frac{1}{n} g(x, y, n)
$$

Thus, small fluctuations are compensated, the tail is more focused to the main location of the fuel and also individual interferences are smoothed. An example for the first preprocessing steps is shown in Fig. 8.

In the next step, the image gray values are inverted to perform a column-wise peak detection and to identify the locations of the fuel. In order to detect these peaks, the topographic property peak prominence (also known as relative height) is considered. Additionally, a minimum prominence value and a minimum peak width are set as further criteria. The coordinates $y_{P, i}$ of the peaks with the highest topographic peak prominences are determined for each image column $i$. In the case of two or more peaks with the same peak prominence (this does not occur in the acquired image data), the topmost peak in the image column is selected. Together with the width $w_{P, i}$ of the detected peak, a column-wise Gaussian distribution $\left(\mu_{0, i}, \sigma_{0, i}\right)$ is derived for the location of the alternative fuel.

$$
\mu_{0, i}=y_{P, i}, \quad \sigma_{0, i}=\frac{w_{P, i}}{6}
$$

Since the majority of the fuel is located within the peak width, it is assumed that for this application the width corresponds approximately to a $6 \sigma$-interval of a Gaussian distribution. This approach replaces a computationally expensive estimation of a Gaussian distribution based on the image column gray values. An example for this approach is shown in Fig. 8(d).

\section{B. Particle detection}

Using the method of Section III-A, a first evaluation of the location of the alternative fuel can be obtained. However, this method only allows a detection of fuel in areas of a compact fuel tail especially close to the burner mouth. To be able to determine the location of the alternative fuel also in areas further away from the burner, a second method aiming at the detection of particles is necessary. An overview of the method for particle detection is shown in Fig. 9.

At first, the input image is cropped to the ROI like in Section III-A (Fig. 10(a)). Since the detection of particles is performed for every single image, the elimination of interferences by time averaging is no longer given. For this reason, an image enhancement using the Retinex algorithm [22] is applied to each image $g(x, y, n)$ (Fig. 10(b)). Thus, high-contrast image areas are emphasized and particles can be detected easier. The contrast-enhanced image $R(x, y, n)$ is convolved with a Laws texture filter $L$ [23] (Fig. 10(c)).

$$
R_{L}(x, y, n)=R(x, y, n) * L
$$

Different texture filters are available to emphasize different image properties. A combination of Level- and Spot-Laws masks proved to be the appropriate filter to detect particles on smooth surfaces. Particle regions appearing dark can be extracted out of the feature image $R_{L}(x, y, n)$ with the help of threshold segmentation (Fig. 10(d)). If the detected regions comply with the predefined property (size $>5$ pixels), the centroids of the regions are included to a hit list $\left(\mathbf{x}_{P D}, \mathbf{y}_{P D}\right)$. Sorting the hit list with respect to the image columns $i$, a Gaussian distribution $\left(\mu_{i}, \sigma_{i}\right)$ for the location of the alternative fuel can be estimated. The centroids $\mathbf{y}_{P D, i}$ of a selected image column $i$ result in

$$
\begin{gathered}
\mathbf{y}_{P D, i}=\left(y_{1}, y_{2}, \ldots, y_{m}\right), \\
\mu_{i}=\frac{1}{m} \sum_{k=1}^{m} y_{k}, \\
\sigma_{i}=\sqrt{\frac{1}{m-1} \sum_{k=1}^{m}\left(\mu_{i}-y_{k}\right)^{2}}
\end{gathered}
$$

with $m$ the number of the detected particles in column $i$. Examples for the different steps of the particle detection method are shown in Fig. 10. 


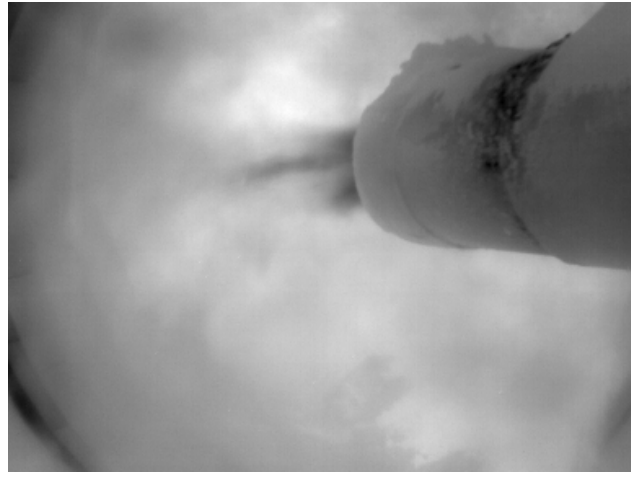

(a)

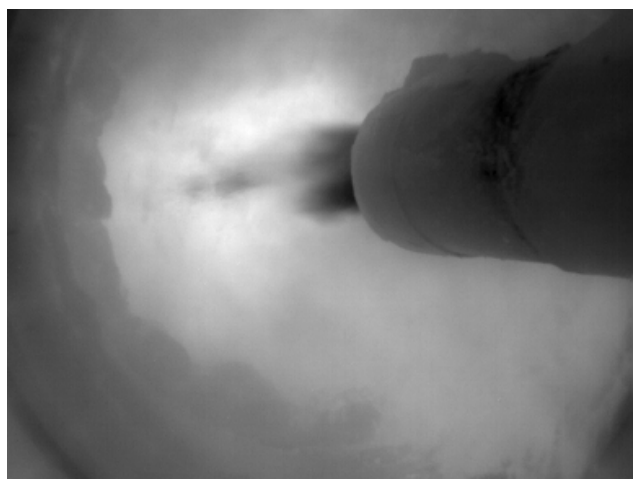

(c)

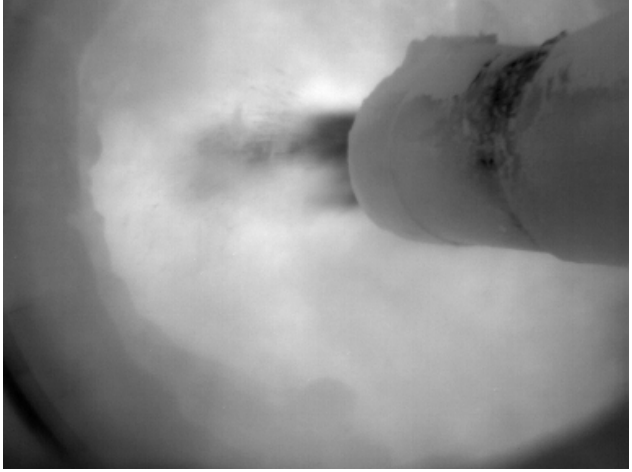

(b)

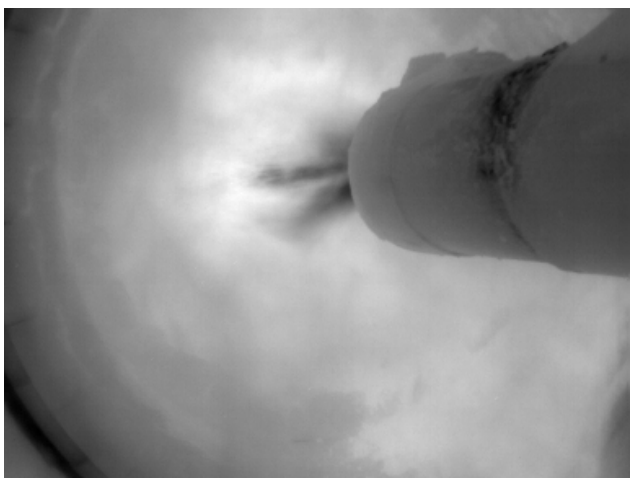

(d)

Fig. 6. Infrared images with different settings of a multi-fuel burner in a rotary kiln of a cement plant. Variation of pneumo deflector with $70 \%$ alternative fuel (a) 40 mbar, (b) 200 mbar and variation of swirl (c) 2 and (d) 8 .

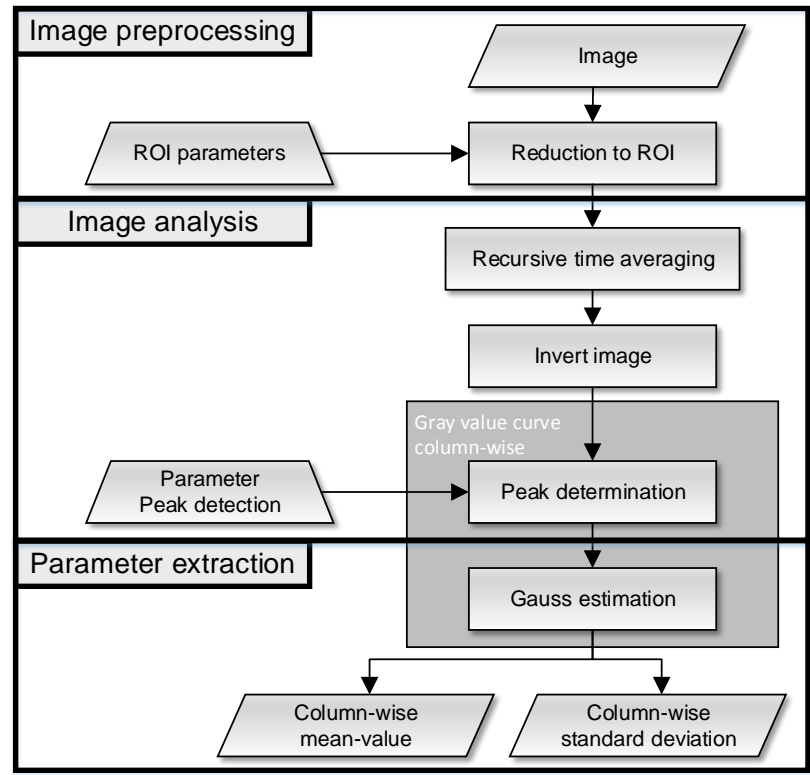

Fig. 7. Flow diagram for the evaluation of the fuel tail close to the burner mouth.

With this method not only particles of alternative fuel are detected. Also dropping material like cakings and other fuel particles are included to the hit list. This leads to false detections, therefore disturbs the location estimation and must be taken into account for further processing.

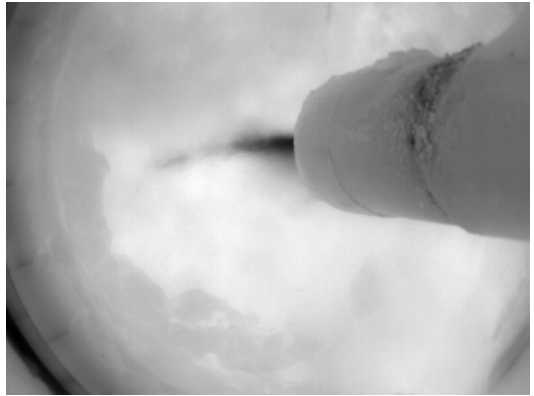

(a)

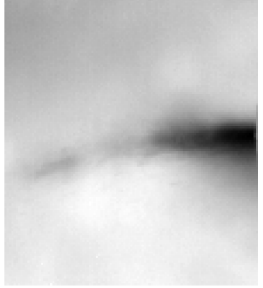

(b)

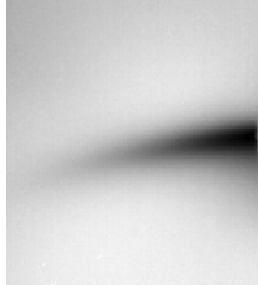

(c)

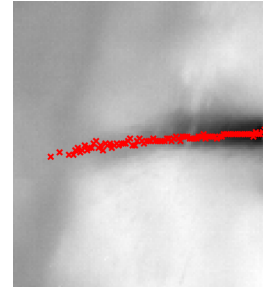

(d)
Fig. 8. Cropping the original image (a) to the region of interest of alternative fuel (ROI) (b), an image after recursive time averaging algorithm (c) and the detection of the average fuel streakline by column-wise gray value peak search $(d)$.

\section{Combining average fuel streakline and particle detection}

The methods of Section III-A and III-B capture the different characteristics of the fuel to determine its location on the 


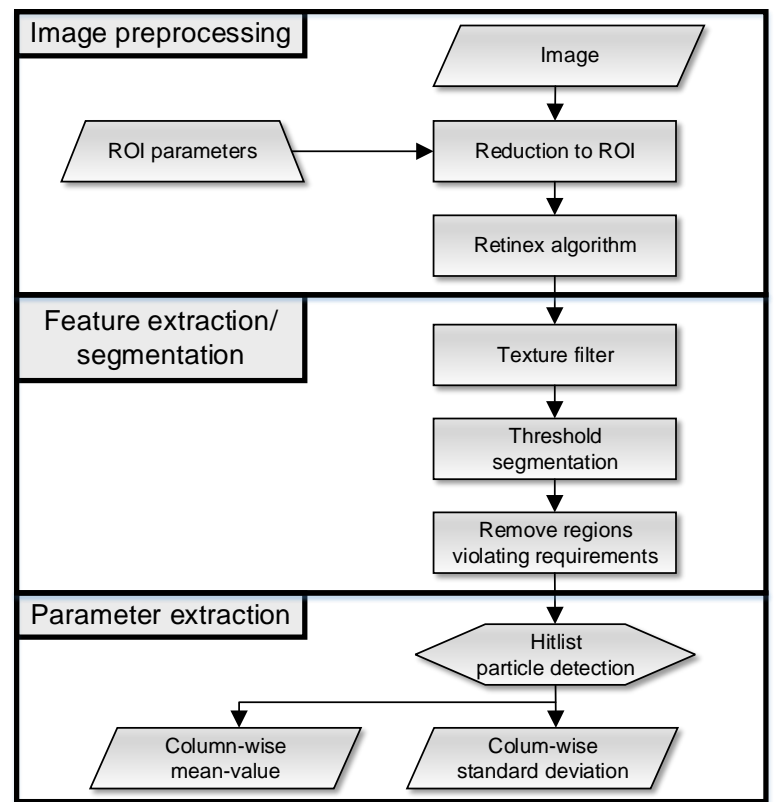

Fig. 9. Flow diagram for the particle detection of alternative fuel.

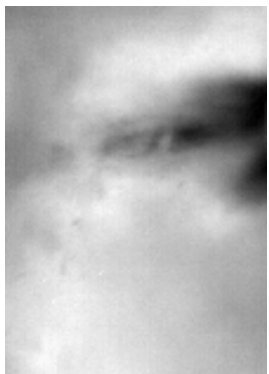

(a)

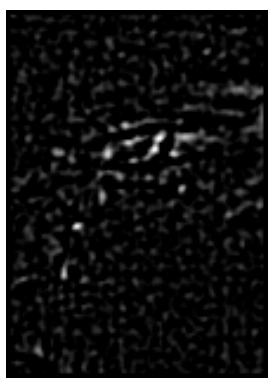

(c)

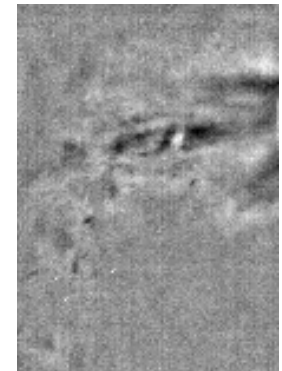

(b)

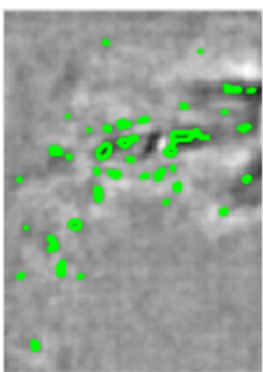

(d)
Fig. 10. Different steps of the particle detection method. (a) Original image cropped to the fuel ROI. (b) Contrast enhancement using Retinex algorithm. (c) Feature image after applying Laws texture filter. (d) Result of a particle detection.

fuel streakline. Both methods provide a column-wise Gauss estimation for the location of the alternative fuel. Depending on the standard deviation of the detection results and the number of detected particles, a maximum a posteriori (MAP) estimation [24] enables a weighted combination of both methods. The combination is performed separately for each image column. The results from the average fuel streakline algorithm of Section III-A $\left(\mu_{0, i}, \sigma_{0, i}\right)$ provide the prior distribution. The distributions of the particle detection $\left(\mu_{i}, \sigma_{i}\right)$ are used to update the estimation. The combined estimation for one image column

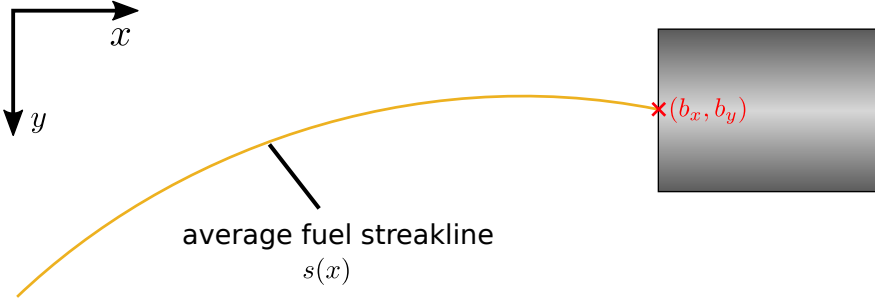

Fig. 11. Geometric constraints for determining the average fuel streakline.

$i$ is received with a MAP estimation

$$
\begin{gathered}
\sigma_{m, i}^{2}=\frac{1}{\frac{m}{\sigma_{i}^{2}}+\frac{1}{\sigma_{0, i}^{2}}} \\
\mu_{m, i}=\sigma_{m, i}^{2}\left(\frac{\mu_{0, i}}{\sigma_{0, i}^{2}}+\frac{m \mu_{i}}{\sigma_{i}^{2}}\right) .
\end{gathered}
$$

A large number of detected particles rises the influence of the particle detection and the average fuel streakline of Section III-A has no longer any influence on the final result. Since the particle detection may also contain outliers and false detections, the influence of particle detection must be decreased by a custom weighting factor $\omega_{P D}$. Also a minimum number $N$ of detections is required to get a statistically sufficient relevance and to enable the particle detection for streakline estimation. Finally, this leads to an adapted MAP estimation

$$
\begin{gathered}
\sigma_{m, i}^{2}=\frac{1}{\frac{m \cdot \omega_{P D} \cdot(m>N)}{\sigma_{i}^{2}}+\frac{1}{\sigma_{0, i}^{2}}} \\
\mu_{m, i}=\sigma_{n, i}^{2}\left(\frac{\mu_{0, i}}{\sigma_{0, i}^{2}}+\frac{m \cdot \omega_{P D} \cdot(m>N) \cdot \mu_{i}}{\sigma_{i}^{2}}\right)
\end{gathered}
$$

and a column-wise estimation of the location of the alternative fuel is received.

\section{Weighted least-squares polynom estimation}

Using the estimates of location $\left(\mu_{m, i}, \sigma_{m, i}\right)$ from Section III-C, the alternative fuel streakline can be estimated through a weighted least-square (WLS) approach. The fuel streakline corresponds to an inclined throw and can be approximated by a second-order polynomial. At constant mounting position of the camera, the outlet of the fuel at the burner mouth can be assumed to be fixed and given. The outlet point $B=\left(b_{x}, b_{y}\right)$ relates to a physical boundary condition for the fuel streakline and thus one parameter of the polynomial can be eliminated. This results in an alternative fuel streakline

$$
s(x)=b_{y}+\theta_{0}\left(x^{2}-b_{x}^{2}\right)+\theta_{1}\left(x-b_{x}\right),
$$

with the parameters $\boldsymbol{\theta}=\left(\theta_{0}, \theta_{1}\right)^{T}$ to be determined (Fig. 11).

Furthermore, the fuel streakline must be left curved because of the assumption of an inclined throw and with respect to the axis direction of the image.

$$
\frac{d^{2} s(x)}{d x^{2}}>0 \Rightarrow \theta_{0}>0
$$


The parameters $\boldsymbol{\theta}$ of the fuel streakline can be determined by a WLS estimation

$$
\widehat{\boldsymbol{\theta}}=\left(\boldsymbol{X}^{T} \boldsymbol{K}^{-1} \boldsymbol{X}\right)^{-1} \boldsymbol{X}^{T} \boldsymbol{K}^{-1} \boldsymbol{\mu},
$$

with

$$
\begin{gathered}
\boldsymbol{X}=\left(\begin{array}{cc}
1^{2}-b_{x}^{2} & 1-b_{x} \\
2^{2}-b_{x}^{2} & 2-b_{x} \\
\vdots & \vdots \\
M^{2}-b_{x}^{2} & M-b_{x}
\end{array}\right), \\
\boldsymbol{K}=\left(\begin{array}{cccc}
\sigma_{m, 1} & 0 & \cdots & 0 \\
0 & \sigma_{m, 2} & \ddots & \vdots \\
\vdots & \ddots & \ddots & \vdots \\
0 & \cdots & \cdots & \sigma_{m, M}
\end{array}\right)
\end{gathered}
$$

and

$$
\boldsymbol{\mu}=\left(\begin{array}{c}
\mu_{m, 1}-b_{y} \\
\mu_{m, 2}-b_{y} \\
\vdots \\
\mu_{m, M}-b_{y}
\end{array}\right),
$$

with $M$ the number of image columns. If the WLS delivers negative values for $\theta_{0}$ and thus violates the physical boundary conditions $\theta_{0}>0, \theta_{0}$ is set to zero and the WLS is performed again based on the fuel streakline

$$
s(x)=b_{y}+\theta_{1}\left(x-b_{x}\right) .
$$

The estimated parameters $\widehat{\boldsymbol{\theta}}=\left(\widehat{\theta_{0}}, \widehat{\theta_{1}}\right)^{T}$ describe the average alternative fuel streakline.

\section{E. Feature extraction}

By means of the average fuel streakline, further image-based parameters can be derived which allow the characterization of the current state of combustion:

- The point of impact of unburned fuel reflects the throw distance of the fuel from the burner mouth to a potential impact on the kiln wall/clinker bed. It can be calculated as the point of intersection of the streakline and a line connecting the two lower points of both kiln ends and by applying a geometric model of the kiln.

- The derivation of the streakline $\frac{d s(x)}{d x}$ close to the burner mouth describes the exit angle of the alternative fuel at the burner mouth.

- The consideration of the column-wise standard deviation $\sigma_{n, i}^{2}$ from Section III-C provides an estimate of the alternative fuel scattering. This scattering can be visualized e.g. by an upper and a lower limit of the alternative fuel in the images.

- The point of combustion can be derived by taking into account the leftmost detection of fuel on the streakline. Based on a geometrical model, the distance from the burner mouth to the point of combustion in world coordinates is computable.

By a continuous monitoring of these parameters, an evaluation of fluctuations in alternative fuel properties and thereby rapid adjustment of the burner are possible.

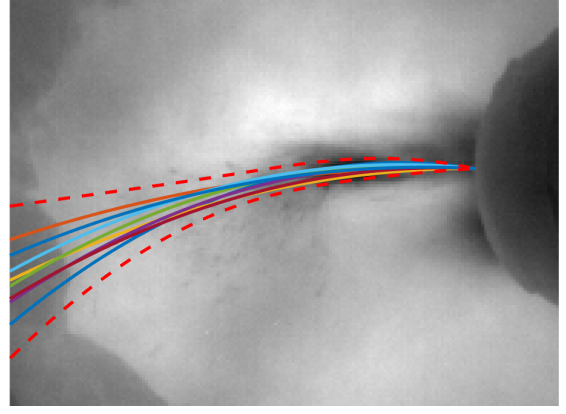

(a)

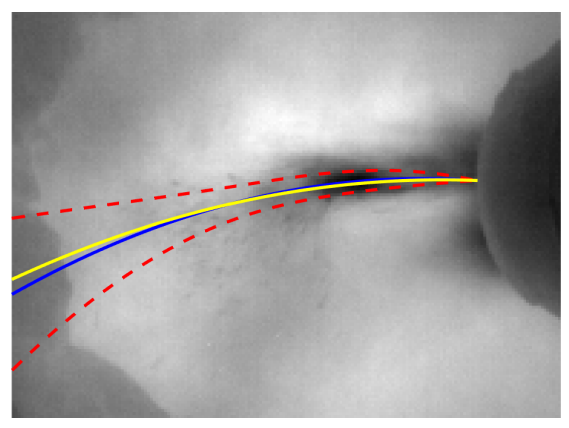

(b)

Fig. 12. Reference data for fuel streakline estimation. (a) solid lines: specified fuel streaklines of experts, dotted lines: $6 \sigma$ interval of experts' data. (b) Comparison of the average fuel streakline of the new methods (yellow) and the reference data (blue).

\section{RESULTS AND DISCUSSION}

Reference or ground truth data to validate the presented methods from Section III are not available, since there is no alternative measurement method that provides information on real flight data of alternative fuel from multi-fuel burners under process conditions. In order to perform a validation of the methods, new reference data are generated using expert knowledge.

\section{A. Generation of reference data}

A software tool has been developed which allows to manually set fuel streaklines into example sequences. Due to the large amount of image data, 56 image sequences during an experiment with varying settings of the multi-fuel burner were selected (each 30 seconds with a time lag of around six minutes between two sequences) and evaluated by eight experts (engineers from cement industry and image processing). For each presented sequence the experts determined the particular streakline manually (supported by the tool). The average fuel streakline over all expert streaklines is used as reference data for each sequence. An example of a fuel streakline from the reference data is shown in Fig. 12.

\section{B. Results compared to reference data}

The deviation between the average alternative fuel streakline from the methods and the reference data is taken as evaluation criterion for the validation of the methods from Section III. The deviation is measured by the size of the area between the two streaklines. A deviation of zero means an exact match of 


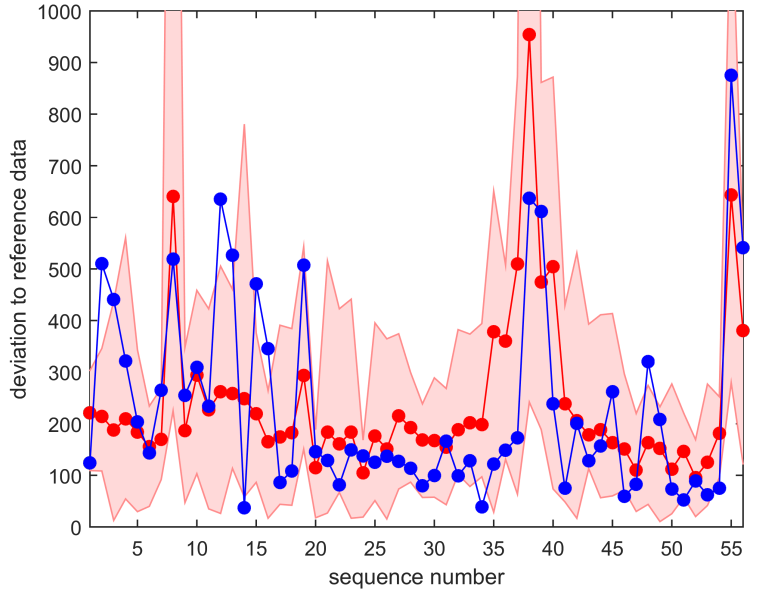

(a)

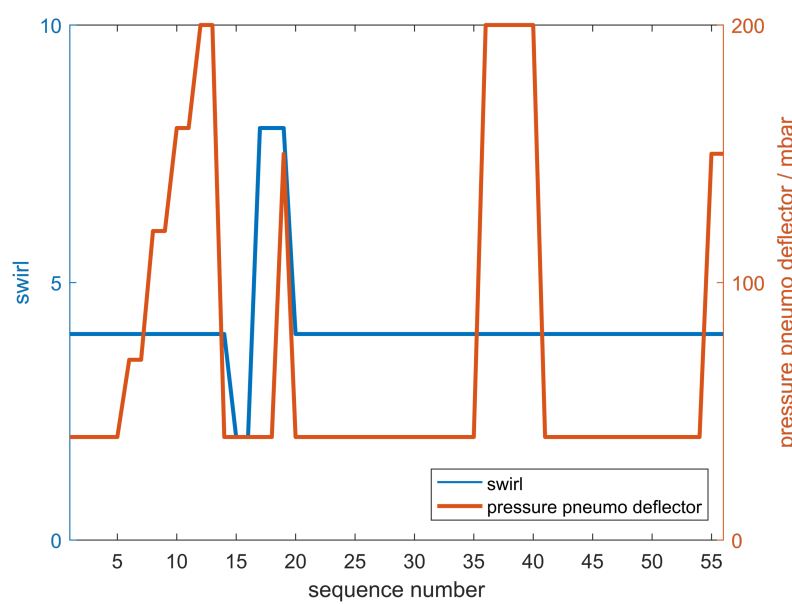

(b)

Fig. 13. (a) Deviation to reference streaklines (area between two streaklines) for automatically detected fuel streaklines (blue) and for the average deviation of all experts' streaklines (red; red area: minimum and maximum deviation in the reference data). The dots reflect the analyzed sequences, the lines interpolate the time between the examined sequences. (b) Burner settings during reference data sequences.

both streaklines, a high deviation value reflects very dissimilar streaklines. Fig. 13(a) shows the comparison with reference data.

The minimum, average and maximum deviation of the fuel streaklines of all experts are used for comparison. $59 \%$ of the automatically detected fuel streaklines from the new methods are within the average deviation of expert evaluation. $89 \%$ are within the maximum deviation of expert evaluation. Table I shows the cumulative deviation over all sequences for reference data, the methods of Section III-A and III-B and the combination of these methods through original and adapted MAP estimation.

\section{Discussion}

Compared to the deviation within the reference data a good estimate of the real fuel streakline is achieved by the method of average fuel streakline. In contrast, the streakline estimated by particle detection differs very clearly from reference data. Although a better result is achieved by combining both meth-
TABLE I

Comparison of the Cumulative DeViation of the DifFerent METHOdS FROM SECTION III AND REFERENCE DATA.

\begin{tabular}{|c||c|}
\hline method & cumulative deviation \\
\hline \hline reference data & 13472.1 \\
\hline average fuel streakline & 14635.8 \\
\hline particle detection & 35253.0 \\
\hline MAP estimation & 20193.3 \\
\hline adapted MAP estimation & 12994.0 \\
\hline
\end{tabular}

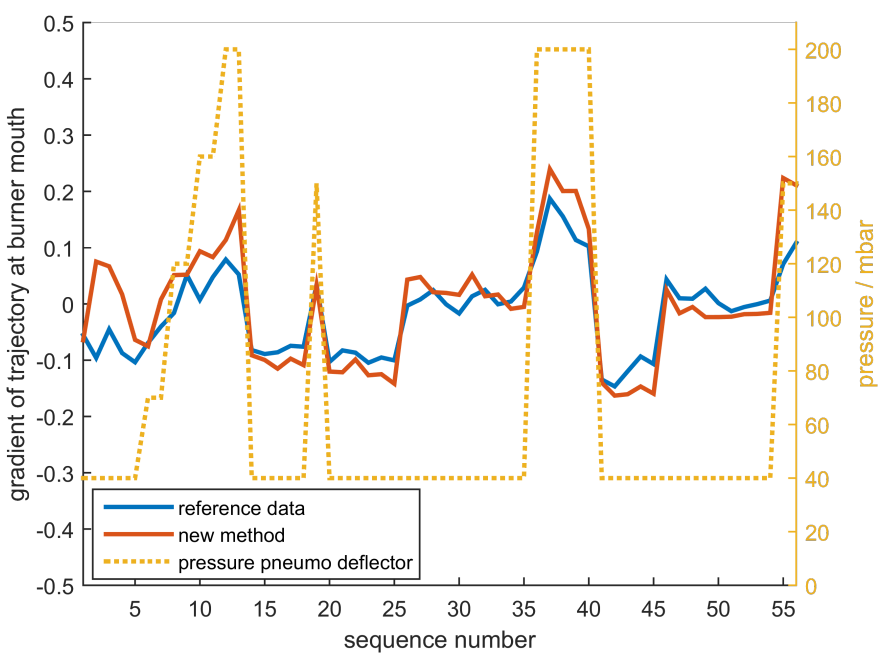

Fig. 14. Comparison of the gradient close to burner mouth of the fuel streaklines from reference data and new methods and the setting of pneumo deflector pressure.

ods with original MAP estimation, it is not better than using the average fuel streakline. The best result with respect to reference data is reached by adapting the MAP estimation with a weighting factor $\omega_{P D}$ as proposed in Section III-C.

A better result is prevented by strong deviations of a few sequences (Fig. 13(a)). Comparing the sequences with strong deviations with the burner settings (Fig. 13(b)), extreme settings for pneumo deflector pressure and swirl can be observed (sequence number 5-20). Especially further away from the burner mouth, a high pneumo deflector pressure leads to stronger scattering of the fuel. Thus, the detection of the average fuel streakline can become difficult and the higher deviation can be explained. Consistently good results are achieved by the automatic detection using the new methods for constant settings (sequence number 21-35 and 40-50).

By derivation of parameters from the detected streaklines, changing burner settings which influence the fuel streakline can be observed. In this case, the change of settings simulates the fluctuating characteristics of the alternative fuel (during the measurement campaign it was not possible to influence the fuel properties). As mentioned in Section III-E, one possible parameter can be the gradient of the fuel streakline $\frac{d s(x)}{d x}$ close to the burner mouth (exit angle). This parameter should increase if the pneumo deflector pressure gets higher. Fig. 14 shows the parameter for fuel streaklines of reference data and automatic detection. The expected trends for varying pneumo deflector pressures are captured.

Strong changes of the parameters at constant burner settings 


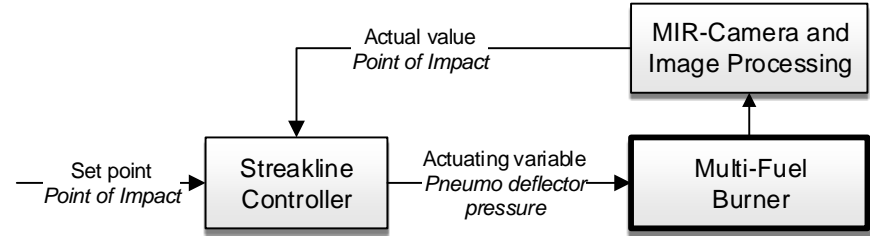

Fig. 15. The image-based feature point of impact in a control concept.

at sequence number 25 can be attributed to changes in the installation position of the camera. Apart from that, differences between automatic detection and reference data are only slightly visible. Overall, the comparison with reference data shows that the automatic detected fuel streaklines correlate very strongly with human perception (expert knowledge) and thus the real fuel flight characteristic. In order to eliminate the influence of the camera position on the extracted image-based features in future installations, a new camera mounting with a traversing unit has been built. This leads to a fixed camera position in the kiln and allows a calibration procedure during the kiln maintenance time.

Depending on the camera system either the alternative fuel (long-wavelength infrared with spectral filter as shown here) or the flame (visual or near-infrared) can be observed. It is not possible to capture both at the same time with the same camera system. In order to analyze a relation between alternative fuel and e.g. the flame shape an additional opening for a camera system (visual or near-infrared) close to the midinfrared camera is required.

The extracted image-based features can be used to increase the process automation by including them into the process control system. Figure 15 shows a possible control framework where the feature point of impact is used as controlled process variable. Based on experience an optimal value of the point of impact can be set by the operator. If deviations from this value occur (e.g. due to changes in fuel properties) the controller adapts the pneumo deflector pressure automatically. The adaption of the fuel composition or the swirl settings based on the feature point of combustion is another possible control framework.

\section{CONCLUSION AND OUTLOOK}

The new camera-based methods for automatically detecting the fuel streakline proposed in this article allow an early detection of changes in the combustion characteristics of alternative fuel. Even under difficult visual conditions as in cement production the detection of the fuel streakline is possible and deviates only slightly from the evaluation of experts.

Based on the fuel streakline new image-based features which describe the current combustion behavior of the alternative fuel are derived. These features allow early counteractions via adaptions of burner settings. Therefore it is possible to facilitate a constant operation with higher fractions of alternative fuels both by supporting the plant operator and by integrating the features into the process control system.

The next step will be a long-term measurement campaign at an industrial plant over several weeks. It will be focused on the analysis of varying alternative fuel properties and on the parameterization and applicability of the process control concepts.

\section{ACKNOWLEDGMENT}

The authors would like to acknowledge the KIC InnoEnergy company for co-funding the research.

\section{REFERENCES}

[1] A. Rahman, M. Rasul, M. Khan, and S. Sharma, "Impact of alternative fuels on the cement manufacturing plant performance: an overview," Procedia Eng., vol. 56, pp. 393-400, 2013.

[2] A. K. Amiruddin and S. Sapuan, "Burner flame image analysis techniques," National Conference on Software Engineering \& Computer Systems (NACES), 2007.

[3] J. Ballester and T. Garcia-Armingol, "Diagnostic techniques for the monitoring and control of practical flames," Prog. Energ. Combust., vol. 36, pp. 375-411, 2010.

[4] W. B. Baek, S. J. Lee, S. Y. Baeg, and C. H. Cho, "Flame image processing and analysis for optimal coal firing of thermal power plant," International Symposium on Industrial Electronics (ISIE), pp. 928-931, 2001.

[5] W.-B. Horng, J.-W. Peng, and C.-Y. Chen, "A new image-based real-time flame detection method using color analysis," International Conference on Networking, Sensing and Control, pp. 100-105, 2005.

[6] C. Duchesne, J. J. Liu, and J. F. MacGregor, "Multivariate image analysis in the process industries: A review," Chemometr. Intell. Lab., vol. 117, pp. 116-128, 2012.

[7] H. Yu and J. F. MacGregor, "Monitoring flames in an industrial boiler using multivariate image analysis," AIChE, vol. 50, no. 7, pp. 14741483, July 2004.

[8] G. Lu, G. Gilabert, and Y. Yan, "Vision based monitoring and characterisation of combustion flames," J. Phys. Conf. Ser. XIII, vol. 15, pp. 194-200, 2005.

[9] H. Chen, X. Zhang, P. Hong, H. Hu, and X. Yin, "Recognition of the temperature condition of a rotary kiln using dynamic features of a series of blurry flame images," IEEE Transactions on Industrial Informatics, vol. 12, no. 1, pp. 148-157, 2016.

[10] J. Smart, G. Lu, Y. Yan, and G. Riley, "Characterisation of an oxy-coal flame through digital imaging," Combust. Flame, vol. 157, pp. 11321139, 2010.

[11] D. Sun, G. Lu, H. Zhou, and Y. Yan, "Flame stability monitoring and characterization through digital imaging and spectral analysis," Meas. Sci. Technol., vol. 22, p. 114007, 2011.

[12] X. Yang, J. Pei, and W. Yang, "Firing particle flow detection and tracking," $3 \mathrm{~d}$ World Congress on Intelligent Control and Automation, pp. 2666-2670, 2000

[13] L. Zhang, E. Binner, Y. Qiao, and C.-Z. Li, "High-speed camera observation of coal combustion in air and $\mathrm{O}_{2} / \mathrm{CO}_{2}$ mixtures and measurement of burning coal particle velocity," Energy Fuels, vol. 24, pp. 29-37, 2010.

[14] J. Matthes, J. Hock, P. Waibel, A. Scherrmann, H.-J. Gehrmann, and H. B. Keller, "A high-speed camera based approach for the on-line analysis of particles in multi-fuel burner flames," Exp. Therm Fluid Sci. vol. 73 , pp. 10-17, 2016

[15] J. Matthes, P. Waibel, and H. B. Keller, "A new infrared camera-based technology for the optimization of the waelz process for zinc recycling," Miner. Eng., vol. 24, no. 8, pp. 944-949, 2011.

[16] — "Detection of empty grate regions in firing processes using infrared cameras," 11th International Conference on Quantitative Infrared Thermography (QIRT), 2012.

[17] P. Waibel, M. Vogelbacher, J. Matthes, and H. B. Keller, "Infrared camera-based detection and analysis of barrels in rotary kilns for waste incineration," 11th International Conference on Quantitative Infrared Thermography (QIRT), 2012.

[18] S. Zipser, A. Gommlich, J. Matthes, H. B. Keller, and C. Fouda, "On the optimization of industrial combustion processes using infrared thermography," 23rd IASTED International Conference on Modelling, Identification and Control, 2004.

[19] R. Mehran, B. E. Moore, and M. Shah, A Streakline Representation of Flow in Crowded Scenes. Berlin, Heidelberg: Springer Berlin Heidelberg, 2010, pp. 439-452. 
[20] Unitherm Cemcon, "M.A.S. Kiln Burner - UNICAL Calciner Burner / M.A.S. GAS Burner," Brochure, 2015.

[21] R. Gade and T. B. Moeslund, "Thermalcamerasd and applications: a survey," Mach. Vis. Appl., vol. 25, 2014.

[22] G. S. Rajput and Z. Rahman, "Hazard detection on runways using image processing techniques," Enhanced and Synthetic Vision (SPIE), 2008.

[23] K. I. Laws, "Textured Image Segmentation," Report No. 940, University of Southern California, USA, 1980.

[24] K. P. Murphy, "Conjugate Bayesian analysis of the Gaussian distribution," University of British Columbia, Tech. Rep., 2007.

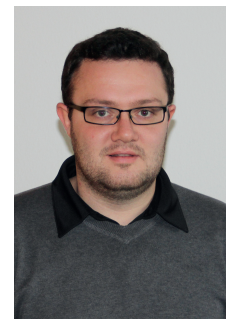

Markus Vogelbacher received the Dipl.-Ing. degree from the Karlsruhe Institute of Technology (KIT), Germany in mechanical engineering in 2011. He works since 2012 as a research staff member at the KIT and since 2015 as a PhD student at the Institute for Applied Computer Science. His research interests include computer vision, signal processing, data analysis and process optimization.

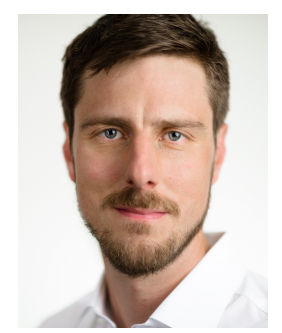

Patrick Waibel received the Dipl.-Ing. degree from the Technical University of Karlsruhe, Germany in electrical engineering in 2007. In 2014 he received the $\mathrm{PhD}$ from the Karlsruhe Institute of Technology (KIT), Germany in mechanical engineering. Since 2008 he works as a research staff member at the Institute for Applied Computer Science at the Karlsruhe Institute of Technology (KIT), Germany. His research interests include computer vision, signal processing, data analysis and process optimization.

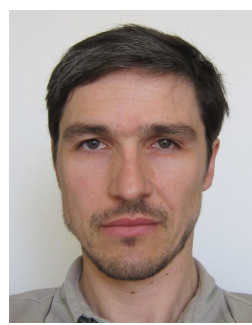

Jörg Matthes received the Dipl.-Ing. degree in mechatronics from the Technical University of Freiberg, Germany in 1999 and the Dr.-Ing. degree from the Technical University of Karlsruhe, Germany in 2004. Since 2000 he is a research staff member at the Institute for Applied Computer Science, Karlsruhe Institute of Technology (KIT), Karlsruhe, Germany. His research interests are in computer vision and process control.

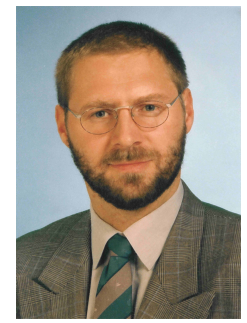

Hubert B. Keller received his Diploma in Engineering Science in 1982 from the University of Karlsruhe and his Doctor of Engineering (Dr.-Ing.) in Applied Computer Science from the University of Clausthal in 1988. After his Study he worked as Development Engineer of Process Control Systems at Siemens AG, Karlsruhe, until 1984 and after then as Scientist and Head of Group at the nuclear research Centre Karlsruhe. At the Karlsruhe Institute of Technology, he is head of the group Process optimization, intelligent sensor systems and nets and safe and secure software. His research interests are Real Time Systems, Software Engineering, Machine Intelligence, intelligent Sensor Systems and Nets and safe and secure Software including Process Control. He is Chairman of Ada Germany e.V. since 1998, Chairman of HybridSensorNet e.V. since 2013 and Chair of Automotive Safety\&Security conferences since 2004. He is Lecturer of Computer Engineering, Faculty for Mechanical Engineering, KIT. 